\title{
Valor Prognóstico dos Critérios de Obesidade em Pacientes Submetidos a Intervenção Coronária Percutânea
}

\author{
José Carlos Estival Tarastchuk¹, Ronaldo da Rocha Loures Bueno , Paulo Maurício Piá de Andrade , \\ Deborah Christina Nercolini ${ }^{1}$, Ênio Eduardo Guérios ${ }^{1}$, João Gustavo Gongora Ferraz ${ }^{2}$
}

\section{RESUMO}

Introdução: Não há critérios de obesidade específicos para a população brasileira. Os pontos de corte preconizados para os sexos feminino e masculino (circunferência abdominal [CA] - $80 \mathrm{~cm}$ e $90 \mathrm{~cm}$, relação cintura-quadril [RCQ] - 0,80 e 0,90, índice de conicidade - 1,18 e 1,25 e índice de massa corporal [IMC] - 30, respectivamente) necessitam ser validados como fatores prognósticos após intervenção coronária percutânea (ICP). Objetivo: O objetivo deste estudo foi verificar os pontos de corte de índices antropométricos da população estudada e compará-los à literatura, quanto à ocorrência de desfechos pós-ICP. Método: Foram incluídos nesta análise 308 pacientes submetidos a ICP, predominantemente do sexo masculino $(60,7 \%)$, com idade de $61,9 \pm 11,1$ anos. Após seis meses, pesquisaram-se os desfechos óbito, infarto agudo do miocárdio, revascularização do vaso-alvo, angina ou prova funcional isquêmica. Os pacientes foram divididos em: Grupo 1 (com desfechos; $\mathrm{n}=91$ ) e Grupo 2 (sem desfechos; $n=217$ ). Para obtenção dos pontos de corte, foram construídas curvas receiver operating characteristic (ROC) dos índices antropométricos versus desfechos. Resultados: Nas mulheres, os pontos de corte encontrados foram: CA, 102 cm; RCQ, 0,93; índice de conicidade, 1,18; e IMC, 24,5. Nos homens, os pontos de corte foram: CA, $102 \mathrm{~cm}$; RCQ, 0,94; índice de conicidade, 1,24; e IMC, 24,98. Comparados à literatura, os valores obtidos de CA e RCQ, para ambos os sexos, ganharam em especificidade, mas perderam em sensibilidade, enquanto o contrário foi observado para o IMC. O índice de conicidade coincidiu com os valores da literatura no sexo feminino; no sexo masculino, porém, aumentou a sensibilidade, mas diminuiu a especificidade. O poder discriminatório foi baixo para todos os índices, que não se mostraram preditores de desfechos no médio prazo pós-ICP. Conclusão: Nesta comparação, os pontos de corte dos índices antropométricos que melhor se correlacionam com desfechos pós-ICP não

\section{SUMMARY}

\section{Prognostic Value of Obesity Anthropometric Indexes in Patients Undergoing Percutaneous Coronary Interventions}

IBackground: No evidence supports obesity cutoff points recommended by literature for the Brazilian population. Some female/male known cutoff points are: waist circumference (WC) $80 / 90 \mathrm{~cm}$, waist-to-hip ratio (WHR) $0.80 / 0.90$, conicity index $(\mathrm{Cl}) 1.18 / 1.25$, body mass index (BMI) 30. These cutoff points need to be validated for the Brazilian population as prognostic after percutaneous coronary intervention $(\mathrm{PCl})$. Objective: To verify cutoff points of obesity anthropometric indexes in this population and to compare them to International Diabetes Federation values for Latin America in determining MACE after PCI. Methods: 308 patients (mean age $61.92 \pm 11.06$ years old, $60.7 \%$ men) undergoing successful $\mathrm{PCl}$. Six months after, patients were contacted for clinical follow-up. MACE included death, acute myocardial infarction, cardiac surgery, reintervention or evidence of myocardial ischemia in a non-invasive test. Patients were divided into 2 groups: group 1 (with MACE, $\mathrm{n}=91$ ), Group 2 (without MACE, $n=217)$. In order to obtain cutoff points, ROC curves were plotted based on anthropometric indexes and MACE. Results: The cutoff points obtained for women were: WC $102 \mathrm{~cm}$, WHR 0.93, CI 1.18 and BMI 24.53. Compared with IDF values, WC and WHR obtained had more specificity $(76.83 \% \times 31.71 \%$ and $43.9 \% \times 7.32 \%)$, BMI had more sensibility $(66.67 \%$ X $20.51 \%)$. For men, the cutoff points were: WC $102 \mathrm{~cm}$, WHR 0.94, Cl 1.24 and BMI 24.98. WC and WHR had more specificity $(69.63 \%$ X $45.19 \%$ and $7.41 \% \times 2.94 \%$ ). BMI and $\mathrm{Cl}$ had more sensibility (65.38\% X 28.85\% and 55.77\% X 53.85\%). Conclusion: Cutoff points of anthropometric indexes of this population that better correlate with MACE are different than the

\footnotetext{
1 Serviço de Hemodinâmica e Cardiologia Intervencionista do Hospital Universitário Evangélico de Curitiba - Curitiba, PR.

2 Hospital da Cruz Vermelha de Curitiba - Curitiba, PR. Correspondência: José Carlos Estival Tarastchuk. Rua Augusto Stelfeld, 1908 - 4o andar - Bigorrilho - Curitiba, PR - CEP 80730-150 E-mail: jestival@cardiol.br

Recebido em: 10/1/2008 • Aceito em: 25/2/2008
} 
coincidiram com os da literatura. Esses pontos de corte, por um lado, ganharam sensibilidade (ou especificidade), mas, por outro, perderam especificidade (ou sensibilidade) e não se mostraram preditores de desfechos no médio prazo pós-ICP. Nossos dados sugerem que, para valor prognóstico, limites específicos para cada população e doença necessitam ser estabelecidos.

DESCRITORES: Obesidade. Antropometria. Angioplastia transluminal percutânea coronária.

A obesidade, doença que já atinge proporções epidêmicas em todo o mundo ${ }^{1,2}$, apresenta estreita relação com morbidade e mortalidade por doenças metabólicas ou cardiovasculares ${ }^{3,4}$. Para o estudo de obesidade, o índice antropométrico mais utilizado atualmente é o índice de massa corporal (IMC), que não define exatamente o local de depósito de gordura. Na verdade, a distribuição de adiposidade parece ter importante significado clínico, uma vez que obesidade central tem sido associada a maior risco cardiometabólico ${ }^{1,5}$. A relação cintura-quadril (RCQ) e a circunferência abdominal (CA) mostram-se mais freqüentemente associadas a eventos cardiovasculares, como infarto agudo do miocárdio (IAM) e óbito, independentemente do IMC e de outros fatores de risco relacionados 6,7 .

Apesar da importância dos índices antropométricos de obesidade central no prognóstico das doenças cardiovasculares, não há critérios específicos para nossa população ${ }^{8}$. Os valores de pontos de corte utilizados para diagnóstico de obesidade central são derivados daqueles utilizados para diagnóstico de síndrome metabólica. Dessa forma, de acordo com o National Cholesterol Education Program, consideram-se alterados valores de CA acima de $102 \mathrm{~cm}$ para homens e RCQ acima de 0,88 para mulheres ${ }^{9}$. Mais recentemente, a International Diabetes Federation preconizou para a população brasileira valores que variam de 0,90 a $0,94^{10}$. Esses valores, no entanto, têm sido questionados quanto à acurácia em definir obesidade central em algumas populações ${ }^{11-15}$. Outros valores de referência para homens e mulheres, como RCQ 0,90 e $0,80^{10}$ e índice de conicidade 1,18 e 1,25 ${ }^{16}$, respectivamente, e mesmo IMC de 30 para ambos os sexos, necessitam ser validados como fatores prognósticos após intervenção coronária percutânea $(I C P)^{17}$. Não há, até o presente momento, publicações que definam o impacto da obesidade central no cenário da Cardiologia Intervencionista.

O objetivo deste trabalho é verificar os pontos de corte dos índices antropométricos da população estudada que melhor se correlacionam com desfechos após ICP e compará-los aos da literatura quanto à ocorrência de desfechos após o procedimento. literature. Our results suggest that, for prognostic information, we need specific anthropometric cutoff points for each population or disease.

DESCRIPTORS: Obesity. Anthropometry. Angioplasty, transluminal, percutaneous coronary.

\section{MÉTODO}

No período de maio de 2005 a setembro de 2006, foram estudados 308 pacientes, $60,7 \%$ do sexo masculino, com média de idade de 61,9 $\pm 11,1$ anos (34 a 88 anos), submetidos a ICP com sucesso e tratados com stents não-farmacológicos. Em todos os participantes da pesquisa realizou-se anamnese habitual, com coleta de informações a respeito dos fatores de risco para doença coronária. Seguiu-se exame físico, com obtenção de peso e altura. O IMC foi calculado pela razão entre o peso e o quadrado da altura $\left(\mathrm{kg} / \mathrm{m}^{2}\right)$. Foi considerado obesidade valor $\geq 30 \mathrm{~kg} / \mathrm{m}^{2}$. A CA foi obtida medindo-se a circunferência na altura da distância média entre a última costela flutuante e a crista ilíaca. O quadril foi medido na altura dos trocânteres femorais. A RCQ foi determinada pela razão entre a $\mathrm{CA}(\mathrm{cm})$ e a circunferência do quadril $(\mathrm{cm})$. O índice de conicidade foi determinado utilizando-se a equação ${ }^{16}$ :

índice de conicidade $=$ cintura $(\mathrm{cm})$

$$
0,109 \times \frac{\sqrt{\text { peso corporal }(\mathrm{kg})}}{\text { estatura }(\mathrm{m})}
$$

As intervenções coronárias foram realizadas pela via femoral com a técnica padrão ${ }^{18}$. Os pacientes foram pré-tratados com a associação de antiagregantes plaquetários, tendo recebido ácido acetilsalicílico 100 mg e ticlopidina $500 \mathrm{mg} / \mathrm{dia}, 48$ horas antes do procedimento, ou clopidogrel $75 \mathrm{mg} / \mathrm{dia}$, iniciado 24 horas antes do procedimento ou em dose de ataque de $300 \mathrm{mg}$, seis horas antes do procedimento. Essa medicação foi mantida por 30 dias após a intervenção. O sucesso do procedimento foi definido como obtenção de estenose residual inferior a $30 \%$, sem ocorrência de eventos clínicos maiores (óbito, IAM ou necessidade de cirurgia de emergência), durante a fase hospitalar. Seis meses após a intervenção, os pacientes foram contatados, na busca dos seguintes desfechos: óbito, IAM, revascularização do vaso-alvo percutânea ou cirúrgica, recorrência de angina ou prova funcional isquêmica. IAM foi diagnosticado na presença de alteração enzimática acompanhada de, pelo menos, um dos seguintes critérios: sintomas compatíveis com isquemia miocárdica, desenvolvimento de onda $\mathrm{Q}$ patológica ao eletrocardiograma ou alteração eletrocardiográfica indicativa 
de sofrimento miocárdico (elevação ou depressão do segmento ST $)^{18}$. Os pacientes foram divididos em Grupo 1 (com desfechos) e Grupo 2 (sem desfechos). Para obtenção dos pontos de corte dos índices antropométricos dos pacientes deste trabalho (em cada sexo) foram construídas curvas ROC (receiver operating characteristic curve) dos índices antropométricos versus ocorrência de desfechos. Os valores de pontos de corte obtidos neste estudo foram comparados quanto à sensibilidade e à especificidade com os valores preconizados na literatura. Para tanto, os índices antropométricos utilizados para essa comparação, para os sexos feminino e masculino, foram, respectivamente: $\mathrm{CA}>80 \mathrm{~cm}$ e $90 \mathrm{~cm}^{10}, \mathrm{RCQ}>0,80$ e $0,90^{10}$, índice de conicidade $>1,18$ e $1,25^{18}$, e IMC $>30^{17}$ para ambos os sexos. Para análise de variáveis dicotômicas, foi utilizado teste exato de Fisher. Para variáveis contínuas, foi utilizado teste $t$ de Student para amostras independentes, com exceção das variáveis lesão pré e pós, que foram analisadas com o teste não-paramétrico de Mann-Whitney.

\section{RESULTADOS}

Foram avaliados 308 pacientes que receberam implante de stent não-farmacológico, com sucesso, entre maio de 2005 e setembro de 2006. De acordo com a ocorrência de desfechos, os pacientes foram divididos em: Grupo 1 (com desfechos; $\mathrm{n}=91 ; 29,5 \%$ ) e Grupo 2 (sem desfechos; $\mathrm{n}=217 ; 70,45 \%$ ). A Figura 1 demonstra a incidência dos desfechos encontrados.

As características da população estudada estão listadas na Tabela 1.

As características clínicas e angiográficas, a taxa de sucesso imediato, a lesão residual e o índice de complicações foram similares entre os grupos.

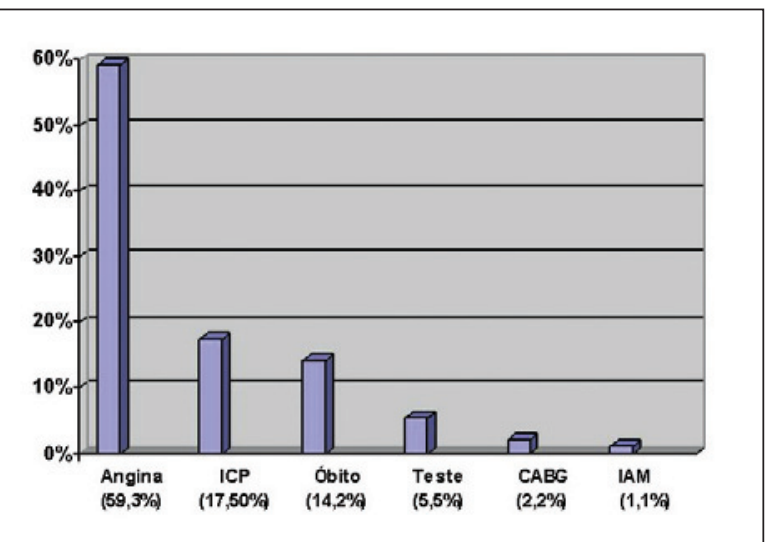

Figura 1 - Incidência de desfechos encontrados na população estudada.

$\mathrm{ICP}=$ intervenção coronária percutânea; óbito = ocorrência de óbito relacionado com insuficiência coronária; Teste = teste não-invasivo positivo para isquemia; $\mathrm{CABG}=$ cirurgia cardíaca vaso-relacionada; $\mathrm{IAM}=$ infarto agudo do miocárdio.

\section{Identificação dos pontos de corte dos índices antropométricos}

Nas mulheres, a área sob a curva ROC da CA para identificar desfechos foi de 0,510 (intervalo de confiança [IC] de $95 \%=0,418-0,602)$. O valor obtido $(102 \mathrm{~cm})$ obteve o melhor equilíbrio entre sensibilidade $(12,8 \%)$ e especificidade $(76,8 \%)$. O valor utilizado pela literatura, de $80 \mathrm{~cm}$, apresentou sensibilidade de 74,4\% e especificidade de 31,7\% (Tabela 2; Figura 2). Para RCQ, a área sob a curva ROC foi de 0,525 (IC 95\% = 0,432$0,616)$. Obteve-se o ponto de corte de 0,93 , com sensibilidade de $43,6 \%$ e especificidade de $43,9 \%$. O valor da literatura, de 0,80 , apresentou $100 \%$ de sensibilidade e 7,3\% de especificidade (Tabela 2; Figura 3). Quanto ao índice de conicidade, a área sob a curva ROC obtida foi de 0,518 (IC 95\%=0,425-0,610). O valor obtido de 1,18 coincidiu com o preconizado pela literatura e teve sensibilidade de $82 \%$ e especificidade de 32,9\% (Tabela 2; Figura 4). Para IMC, a área sob a curva ROC foi de 0,520 (IC 95\% = 0,427-0,612). Para um valor obtido de 24,5 , a sensibilidade obtida foi de $66,7 \%$ e a especificidade, de $24,4 \%$. O IMC de 30 preconizado pela literatura teve sensibilidade de 20,5\% e especificidade de 76,8\% (Tabela 2; Figura 5).

Nos homens, a área sob a curva ROC da CA para identificar desfechos foi de 0,509 (IC 95\% = 0,435$0,583)$. O valor de $102 \mathrm{~cm}$ obteve o melhor equilíbrio entre sensibilidade $(21,15 \%)$ e especificidade $(69,63 \%)$ para predizer desfechos. Para a mesma população, foram obtidas a sensibilidade e a especificidade para a predição de desfechos, com base no valor preconizado pela literatura $(90 \mathrm{~cm}): 57,7 \%$ e $45,2 \%$, respectivamente (Tabela 3; Figura 6). Para RCQ, a área sob

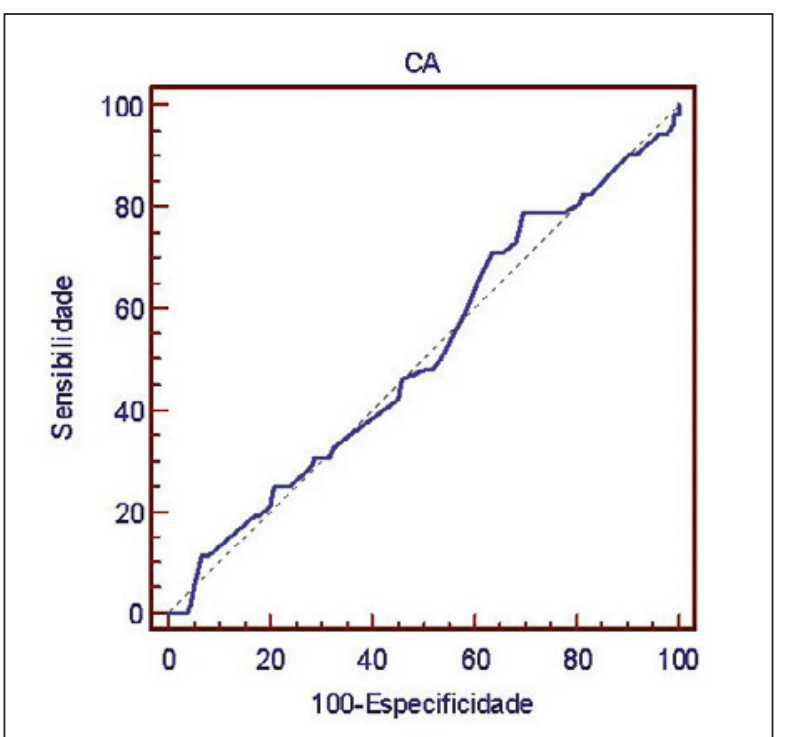

Figura 2 - Curva ROC (receiver operating characteristic curve) da circunferência abdominal para identificar desfecho (sexo feminino). $\mathrm{CA}=$ circunferência abdominal. 
Tarastchuk JCE, et al. Valor Prognóstico dos Critérios de Obesidade em Pacientes Submetidos a Intervenção Coronária Percutânea. Rev Bras Cardiol Invas. 2008;16(1):50-58.

a curva ROC foi de 0,609 (IC 95\%=0,535-0,679). O valor obtido de 0,94 obteve $67,3 \%$ de sensibilidade e $7,4 \%$ de especificidade. O valor da literatura para esse índice antropométrico de 0,90 apresentou sensibilidade especificidade de $86,5 \%$ e $2,9 \%$, respectivamente (Tabela 3; Figura 7). Para o índice de conicidade, a área sob a curva ROC foi de 0,514 (IC 95\% = 0,440$0,588)$. O valor obtido de 1,24 obteve sensibilidade de $55,8 \%$ e especificidade de $37 \%$. O valor da litera-

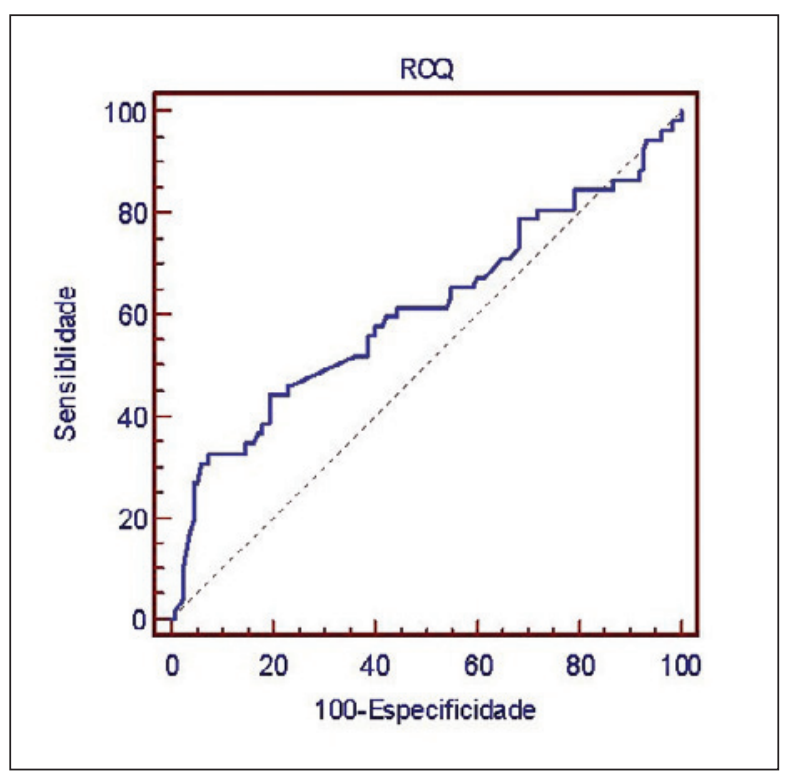

Figura 3 - Curva ROC (receiver operating characteristic curve) da relação cintura-quadril para identificar desfecho (sexo feminino). $\mathrm{RCQ}$ = relação cintura-quadril.



Figura 4 - Curva ROC (receiver operating characteristic curve) do índice de conicidade para identificar desfecho (sexo feminino). $\mathrm{IC}=$ índice de conicidade. tura, de 1,25, apresentou sensibilidade e especificidade para predizer desfechos nesse grupo estudado de 53,8\% e 38,5\%, respectivamente (Tabela 3; Figura 8). No que diz respeito ao IMC, a área sob a curva ROC encontrada foi de 0,503 (IC 95\% =0,429-0,577). O valor obtido de 24,98 esteve associado a sensibilidade de $65,4 \%$ e especificidade de $26,7 \%$. O IMC de 30 preconizado pela literatura teve sensibilidade de $28,8 \%$ e especificidade de 71,1\% (Tabela 3; Figura 9).

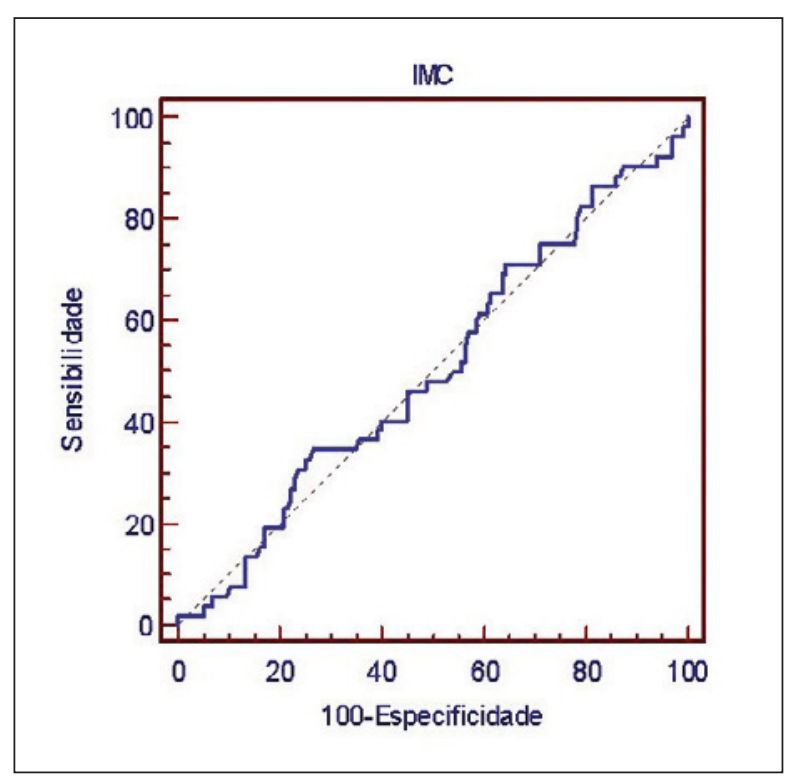

Figura 5 - Curva ROC (receiver operating characteristic curve) do índice de massa corporal para identificar desfecho (sexo feminino). IMC = índice de massa corporal.

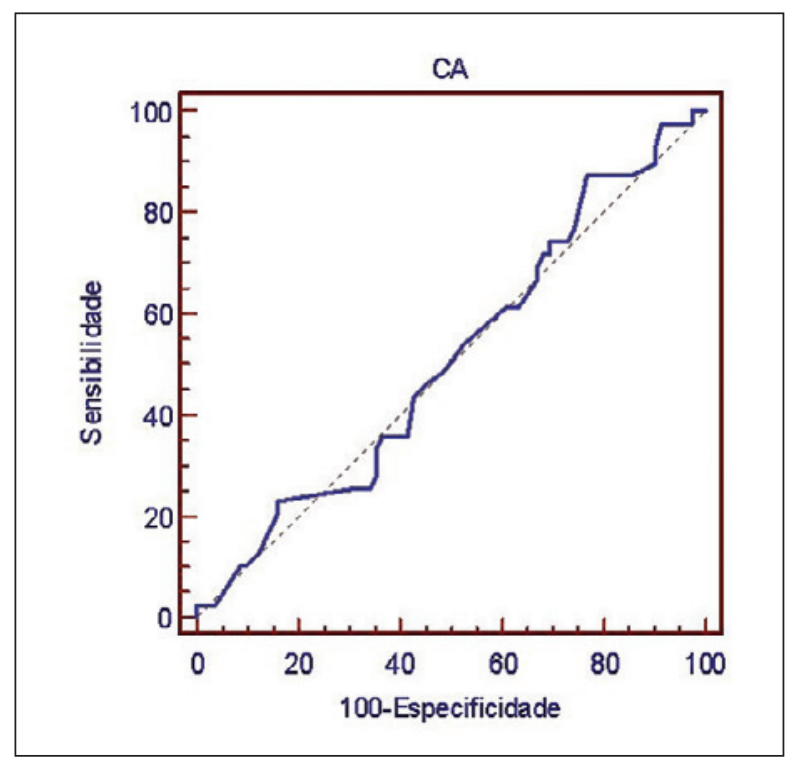

Figura 6 - Curva ROC (receiver operating characteristic curve) da circunferência abdominal para identificar desfecho (sexo masculino). $\mathrm{CA}=$ circunferência abdominal. 


\section{DISCUSSÃo}

Neste estudo, os pontos de corte de índices antropométricos que, de acordo com a curva ROC, melhor se relacionaram com a ocorrência de desfechos após ICP foram, para mulheres e homens, respectivamente: CA, $102 \mathrm{~cm}$ e $102 \mathrm{~cm}$; RCQ, 0,93 e 0,94; índice de conicidade, 1,18 e 1,24; IMC, 24,53 e 24,98. Ao comparar os pontos de corte dos índices antropométricos obtidos no sexo feminino aos preconizados pela literatura, no que diz respeito à ocorrência de desfechos, CA e RCQ ganharam em especificidade $(76,8 \%$ vs. $31,7 \%$ e $43,9 \%$ vs. $7,3 \%$ ), mas perderam em sensibilidade ( $12,8 \%$ vs. $74,4 \%$ e $43,6 \%$ vs. $100 \%)$. Já o valor obtido para o IMC aumentou a sensibilidade $(66,7 \%$ vs. $20,5 \%)$ e diminuiu a especificidade $(24,4 \%$ vs. $76,8 \%)$. Apenas o índice de conicidade nas muIheres coincidiu com o valor apresentado na literatura. Para o sexo masculino, CA e RCQ ganharam em especificidade $(69,6 \%$ vs. $45,2 \%$ e $7,4 \%$ vs. $2,9 \%)$, mas perderam em sensibilidade $(21,1 \%$ vs. $57,7 \%$ e $67,3 \%$ vs. $86,5 \%$ ), enquanto o IMC e o índice de conicidade ganharam em sensibilidade $(65,4 \%$ vs. $28,8 \%$ e $55,8 \%$ vs. $53,8 \%)$, mas perderam em especificidade $(26,7 \%$ vs. $71,1 \%$ e $37 \%$ vs. $38,5 \%$ ).

Para todos os índices, tanto para homens como para mulheres, o poder discriminatório foi baixo, o que é evidenciado pelas áreas sob as curvas ROC próximas a $0,5 \mathrm{ou}$, de forma equivalente, pela proximidade das curvas com a linha da identidade (linha tracejada). Isso significa que, na amostra deste estudo, nenhum índice antropométrico se mostrou preditor de desfechos a médio prazo pós-ICP.

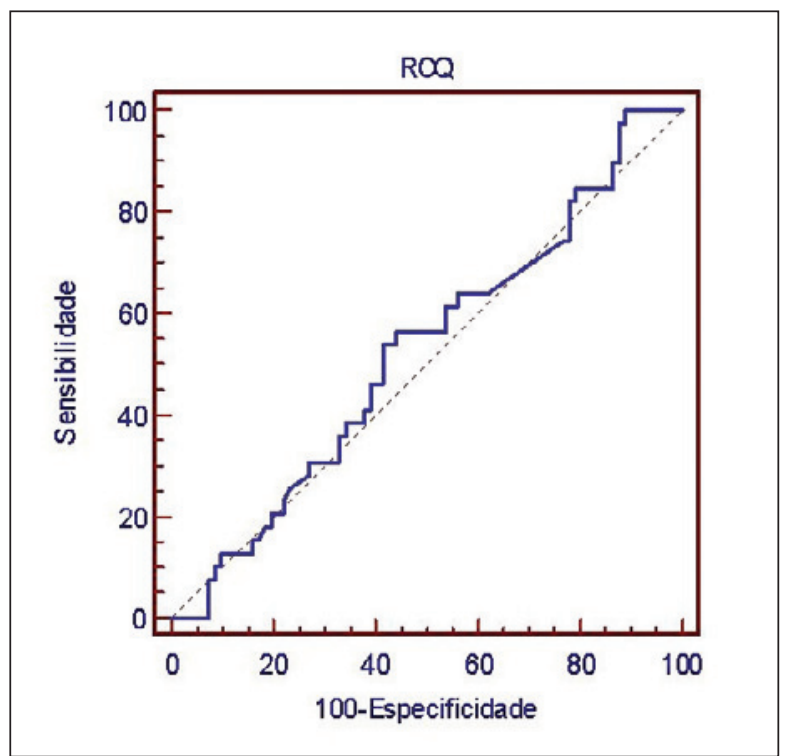

Figura 7 - Curva ROC (receiver operating characteristic curve) da relação cintura-quadril para identificar desfecho (sexo masculino). RCQ = relação cintura-quadril.
Não há, até o presente momento, critérios de obesidade específicos para a população brasileira $^{8}$, nem estudos populacionais associando pontos de corte de obesidade central e desfechos após ICP. Na literatura, encontramos apenas valores para diagnóstico de síndrome metabólica e para predição de risco cardiovascular (Tabela 4). Dentre as publicações que estabelecem pontos de corte para diagnóstico de síndrome metabólica, aqueles estabelecidos pelo National Cholesterol Education Program e pelo National Heart,

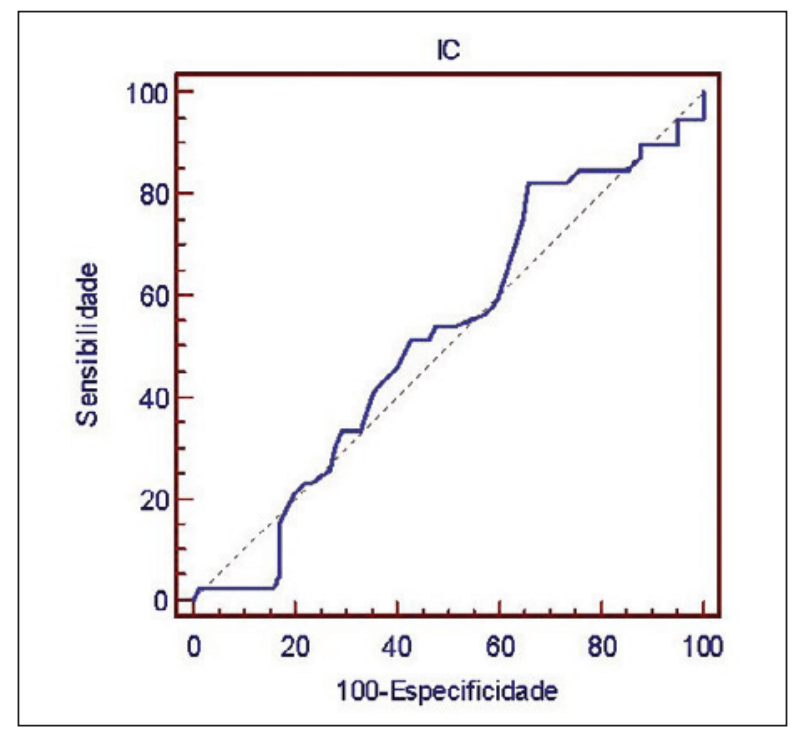

Figura 8 - Curva ROC (receiver operating characteristic curve) do índice de conicidade para identificar desfecho (sexo masculino). $\mathrm{IC}=$ índice de conicidade.

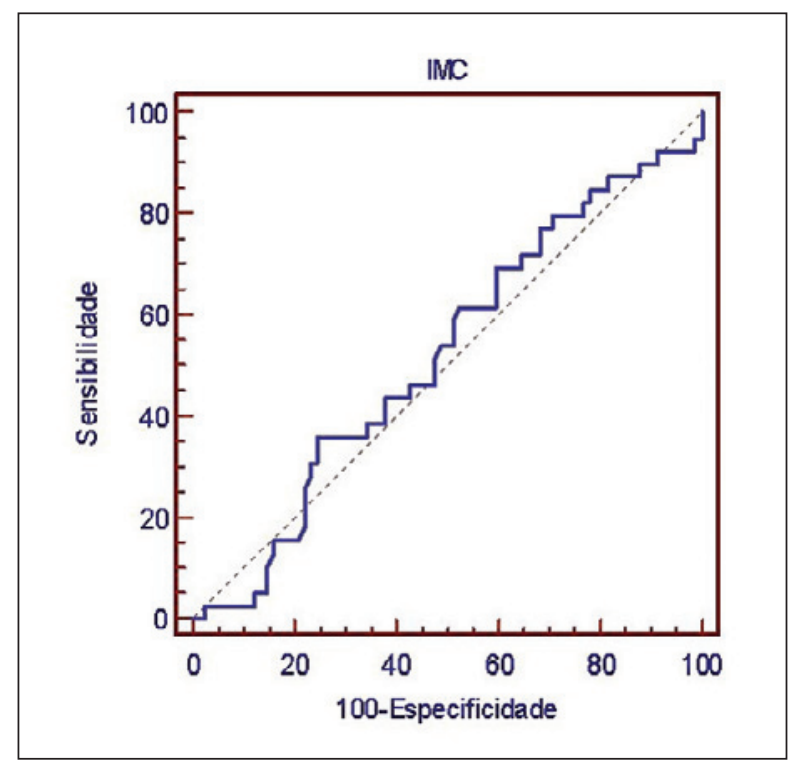

Figura 9 - Curva ROC (receiver operating characteristic curve) do índice de massa corporal para identificar desfecho (sexo masculino). IMC = índice de massa corporal. 
Tarastchuk JCE, et al. Valor Prognóstico dos Critérios de Obesidade em Pacientes Submetidos a Intervenção Coronária Percutânea. Rev Bras Cardiol Invas. 2008;16(1):50-58.

TABELA 1

Características clínicas e angiográficas dos pacientes estudados

\begin{tabular}{|c|c|c|c|}
\hline & $\begin{array}{l}\text { Grupo } 1 \\
(n=91)\end{array}$ & $\begin{array}{c}\text { Grupo } 2 \\
(n=217)\end{array}$ & $\mathbf{p}$ \\
\hline Idade (anos) & $60,6 \pm 11,6$ & $62,5 \pm 10,8$ & 0,19 \\
\hline Sexo masculino & $52(57,1 \%)$ & $135(62,2 \%)$ & 0,44 \\
\hline Baixa escolaridade & $52(57,1 \%)$ & $122(56,2 \%)$ & 0,90 \\
\hline IMC & $27,6 \pm 4,7$ & $27,5 \pm 4,4$ & 0,91 \\
\hline $\mathrm{CA}(\mathrm{cm})$ & $92,1 \pm 13,4$ & $92,8 \pm 13,1$ & 0,70 \\
\hline Quadril (cm) & $93,4 \pm 10,1$ & $91,3 \pm 12,1$ & 0,15 \\
\hline RCQ & $0,99 \pm 0,12$ & $1,02 \pm 0,12$ & 0,08 \\
\hline Índice de conicidade & $1,26 \pm 0,12$ & $1,26 \pm 0,11$ & 0,94 \\
\hline HAS & $72(79,1 \%)$ & $160(73,7 \%)$ & 0,39 \\
\hline DM & $36(39,6 \%)$ & $66(30,4 \%)$ & 0,14 \\
\hline Tabagismo & $20(22 \%)$ & $45(20,7 \%)$ & 0,88 \\
\hline Hipercolesterolemia & $46(50,6 \%)$ & $99(45,6 \%)$ & 0,45 \\
\hline Sedentarismo & $53(58,2 \%)$ & $118(54,4 \%)$ & 0,62 \\
\hline Estresse & $22(24,2 \%)$ & $73(33,6 \%)$ & 0,11 \\
\hline Angina instável & $26(28,6 \%)$ & $60(27,6 \%)$ & 0,89 \\
\hline Angioplastia primária & $9(9,89 \%)$ & $12(5,52 \%)$ & 0,21 \\
\hline ATC DA & $32(39,6 \%)$ & $88(40,6 \%)$ & 0,90 \\
\hline Lesão B2/C & $73(80,2 \%)$ & $166(76,5 \%)$ & 0,55 \\
\hline Lesão em terço inicial do vaso & $43(47,2 \%)$ & $126(58,1 \%)$ & 0,10 \\
\hline Diâmetro $(\mathrm{mm})$ & $2,96 \pm 0,74$ & $2,92 \pm 0,47$ & 0,64 \\
\hline Extensão (mm) & $16,1 \pm 6,1$ & $17,0 \pm 6,3$ & 0,25 \\
\hline Lesão pré (\%) & $86,9 \pm 10,6$ & $84,5 \pm 10,8$ & 0,06 \\
\hline Lesão pós (\%) & $2,6 \pm 6,1$ & $3,6 \pm 8,1$ & 0,44 \\
\hline
\end{tabular}

TABELA 2

Desempenho dos pontos de corte dos índices antropométricos obtidos na população do estudo e preconizados pela literatura na identificação de desfechos após angioplastia transluminal coronária (sexo feminino)

\begin{tabular}{llcccc}
\hline $\begin{array}{l}\text { Índice } \\
\text { antropométrico }\end{array}$ & Ponto de corte & Sensibilidade & Especificidade & $\begin{array}{c}\text { Área sob a } \\
\text { curva ROC }\end{array}$ & IC 95\% \\
\hline IMC & $\geq 30$ (literatura) & $20,5 \%$ & $76,8 \%$ & - & - \\
& $\geq 24,53$ (ROC) & $66,7 \%$ & $24,4 \%$ & 0,520 & $0,427-0,612$ \\
CA & $>80 \mathrm{~cm}$ (literatura) & $74,4 \%$ & $31,7 \%$ & - & - \\
& $>102 \mathrm{~cm}$ (ROC) & $12,8 \%$ & $76,8 \%$ & 0,510 & - \\
RCQ & $>0,80$ (literatura) & $100 \%$ & $7,3 \%$ & - & $0,418-0,602$ \\
& $>0,9382$ (ROC) & $43,6 \%$ & $43,9 \%$ & 0,525 & - \\
Índice de conicidade & $>1,18$ (literatura) & $82 \%$ & $32,9 \%$ & & 0,616 \\
& $>1,18$ (ROC) & $82 \%$ & $32,9 \%$ & 0,518 & $0,425-0,610$ \\
\hline
\end{tabular}

$\mathrm{ROC}=$ receiver operating characteristic curve; $\mathrm{IMC}=$ índice de massa corporal; $\mathrm{CA}=$ circunferência abdominal; $\mathrm{RCQ}=$ relação cintura-quadril; IC = intervalo de confiança. 
TABELA 3

Desempenho dos pontos de corte dos índices antropométricos obtidos na população do estudo e preconizados pela literatura na identificação de desfechos após angioplastia transluminal coronária (sexo masculino)

\begin{tabular}{llcccc}
\hline Índice antropométrico & Ponto de corte & Sensibilidade & Especificidade & Área sob a curva ROC & IC 95\% \\
\hline IMC & $\geq 30$ (literatura) & $28,8 \%$ & $71,1 \%$ & - & - \\
& $\geq 24,98$ (ROC) & $65,4 \%$ & $26,7 \%$ & 0,503 & $0,429-0,577$ \\
CA & $>90 \mathrm{~cm}$ (literatura) & $57,7 \%$ & $45,2 \%$ & - & - \\
& $>102$ (ROC) & $21,1 \%$ & $69,6 \%$ & 0,509 & $0,435-0,583$ \\
RCQ & $>0,90$ (literatura) & $86,5 \%$ & $2,9 \%$ & - & - \\
Índice de conicidade & $>0,9496$ (ROC) & $67,3 \%$ & $7,4 \%$ & 0,609 & $0,535-0,679$ \\
& $>1,25$ (literatura) & $53,8 \%$ & $38,5 \%$ & & $0,440-0,588$ \\
\hline
\end{tabular}

$\mathrm{ROC}=$ receiver operating characteristic curve; $\mathrm{IMC}=$ índice de massa corporal; $\mathrm{CA}=$ circunferência abdominal; $\mathrm{RCQ}=$ relação cintura-quadril; IC = intervalo de confiança.

TABELA 4

Pontos de corte para índices de obesidade encontrados na literatura

\begin{tabular}{|c|c|c|c|c|c|c|c|c|c|c|}
\hline \multirow[t]{2}{*}{ Autor } & \multirow[t]{2}{*}{ Periódico } & \multirow[t]{2}{*}{ Ano } & \multirow[t]{2}{*}{ Origem } & \multirow[t]{2}{*}{ Investigação } & \multicolumn{3}{|c|}{ Sexo feminino } & \multicolumn{3}{|c|}{ Sexo masculino } \\
\hline & & & & & CA & RCQ & IMC & CA & RCQ & IMC \\
\hline $\mathrm{NCEP}^{19}$ & JAMA & 2001 & Estados Unidos & SM & 88 & & & 102 & & \\
\hline Hu et al. ${ }^{20}$ & Arch Int Med & 2004 & Europa & SM & 80 & & & 94 & & \\
\hline Oh et al. ${ }^{21}$ & Diabetes Care & 2004 & Coréia & SM & 80 & & & 94 & & \\
\hline NHLBI ${ }^{15}$ & Circulation & 2005 & Estados Unidos & SM & 80 & & & 85 & & \\
\hline Tan et al. ${ }^{11}$ & Diabetes Care & 2004 & Ásia & SM & 80 & & & 90 & & \\
\hline Ford et al. ${ }^{10}$ & Diabetes Care & 2005 & América Latina & SM & 80 & 0,80 & & 90 & 0,90 & \\
\hline $\begin{array}{l}\text { Diretriz } \\
\text { Brasileira SM²2 }\end{array}$ & Arq Bras Cardiol & 2005 & Brasil & SM & $80-88$ & & & $94-102$ & & \\
\hline Barbosa et al. ${ }^{8}$ & Arq Bras Cardiol & 2006 & Brasil & SM & 84 & & & 88 & & \\
\hline Rexrode et al. ${ }^{23}$ & JAMA & 1998 & Estados Unidos & Risco DAC & 96,5 & 0,88 & & & & \\
\hline Bei-Fan et al. ${ }^{24}$ & $\begin{array}{l}\text { Asia Pac J } \\
\text { Clin Nutr }\end{array}$ & 2002 & China & Risco CV & 80 & & 24 & 85 & & 24 \\
\hline Lin et al..$^{25}$ & $\begin{array}{c}\text { Int J Obes } \\
\text { Relat Metab Dis }\end{array}$ & 2002 & Tailândia & Risco CV & 71,5 & 0,76 & 22,1 & 80,5 & 0,85 & 23,6 \\
\hline $\begin{array}{l}\text { Snehalatha } \\
\text { et al. } .^{26}\end{array}$ & $\begin{array}{l}\text { Diabetes } \\
\text { Care }\end{array}$ & 2003 & Índia & Risco CV & 80 & 0,81 & & 85 & 0,88 & \\
\hline Ito et al. ${ }^{27}$ & $\begin{array}{l}\text { Int J Obes } 2003 \\
\text { Relat Metab Dis }\end{array}$ & Japão & Risco CV & 72 & 0,80 & 22,5 & 84 & 0,90 & 23,5 & \\
\hline $\begin{array}{l}\text { Pitanga \& } \\
\text { Lessa }^{16}\end{array}$ & Arq Bras Cardiol & 2005 & Brasil & Risco CV & 88 & & & 83 & & \\
\hline
\end{tabular}

$\mathrm{CA}=$ circunferência abdominal; $\mathrm{RCQ}=$ relação cintura-quadril; IMC = índice de massa corporal; SM = síndrome metabólica; DAC = doença arterial coronária; $\mathrm{CV}=$ cardiovascular.

Lung, and Blood Institute para a CA de $88 \mathrm{~cm}$ para mulheres e de $102 \mathrm{~cm}$ para homens têm sido questionados por não se adequarem a populações de diferentes etnias ${ }^{8,15,19,22}$. Ainda para diagnóstico dessa doença, a Diretriz Brasileira de Diagnóstico e Tratamento da Síndrome Metabólica preconiza valores de
CA entre $80 \mathrm{~cm}$ e $88 \mathrm{~cm}$ para mulheres e entre $94 \mathrm{~cm}$ e $102 \mathrm{~cm}$ para homens ${ }^{22}$. Em 2004, Hu et al. ${ }^{20}$ (população européia) e Oh et al. ${ }^{21}$ (população coreana) definiram para CA valores normais de até $80 \mathrm{~cm}$ para mulheres e de até $94 \mathrm{~cm}$ para homens. Estudando populações asiáticas, Tan et al. ${ }^{11}$ concluíram que va- 
lores mais corretos para diagnóstico de síndrome metabólica seriam de $80 \mathrm{~cm}$ para mulheres e de $90 \mathrm{~cm}$ para homens, o que coincide com as medidas atualmente indicadas pela International Diabetes Federation para algumas regiões do Brasil ${ }^{10}$. Mais recentemente, Barbosa et al. ${ }^{8}$, estudando a síndrome metabólica na população brasileira, citam medidas de CA de $84 \mathrm{~cm}$ para mulheres e de $88 \mathrm{~cm}$ para homens.

Associando risco cardiovascular e medidas antropométricas de obesidade, Rexrode et al. ${ }^{23}$, em 1998, estipularam, para mulheres, valores para CA de 96,5 cm e para RCQ de 0,88. Ainda no cenário de índices antropométricos e risco cardiovascular, para a população tailandesa, verificaram-se como valores de corte $71,5 \mathrm{~cm}, 0,76$ e 22,1, respectivamente, para CA, RCQ e IMC, no sexo feminino. Para homens, os valores encontrados foram de $80,5 \mathrm{~cm}, 0,85$ e 23,6, respectivamente ${ }^{25}$. Chineses estudados por Bei-Fan ${ }^{24}$, em 2002, apresentaram maior risco de desenvolvimento de doenças cardiovasculares quando a CA era maior que $80 \mathrm{~cm}$ e o IMC era superior a 24 no sexo feminino, e quando a CA era maior que $85 \mathrm{~cm}$ e o IMC era superior a 24 no sexo masculino. Ainda para asiáticos (Japão), o risco cardíaco, segundo Ito et al. ${ }^{27}$, seria maior para mulheres com CA, RCQ e IMC acima de $72 \mathrm{~cm}, 0,80$ e 22,5, respectivamente. Já nos homens, os valores seriam de $84 \mathrm{~cm}, 0,90$ e 23,5, respectivamente. Encontramos, na literatura, valores para população indiana que estariam sob maior risco cardiovascular. Esses valores seriam, para mulheres, maiores que $80 \mathrm{~cm}$ para CA e 0,81 para RCQ e nos homens, $85 \mathrm{~cm}$ para CA e 0,88 para RCQ ${ }^{26}$. Pitanga \& Lessa ${ }^{16}$ obtiveram pontos de corte para amostra de população brasileira. Segundo esses autores, valores de CA maior que $83 \mathrm{~cm}$ para mulheres e de CA maior que $88 \mathrm{~cm}$ para homens estariam relacionados com maior risco cardíaco.

\section{Limitações do estudo}

Por se tratar de uma amostra populacional composta de pacientes consecutivos submetidos a ICP, a maioria apresentou obesidade central, o que pode explicar a pouco expressiva área sob a curva ROC e o conseqüente menor poder discriminatório dos indicadores de obesidade para desfechos. O número de indivíduos da amostra deve ser levado em conta na interpretação dos resultados, devendo o leitor aguardar estudos maiores para confirmação das hipóteses levantadas.

\section{CONCLUSÃO}

Os pontos de corte dos índices antropométricos da população estudada que melhor se correlacionam com desfechos após ICP não coincidem com os encontrados na literatura. Nesta comparação, os pontos de corte obtidos pelas curvas ROC, por um lado, aumentam a sensibilidade (ou a especificidade), mas, por outro, diminuem a especificidade (ou a sensibilidade) e não se mostraram preditores de desfechos a médio prazo pós-ICP. Nossos dados sugerem que, para valor prognóstico, devem ser estabelecidos pontos de corte de índices antropométricos para cada população e doença.

\section{REFERÊNCIAS BIBLIOGRÁFICAS}

1. World Health Organization. Obesity: preventing and managing the global epidemic. Report of a World Health Organization Consultation. Geneva, Switzerland: World Health Organization; 2000. p.256. WHO Obesity Technical Report Series, n.894.

2. Wang $\mathrm{Y}$, Monteiro C, Popkin BM. Trends of obesity and underweight in older children and adolescents in the United States, Brazil, China and Russia. Am J Clin Nutr. 2002;75(6): 971-7.

3. Calle EE, Rodriguez C, Walker-Thurmond K, Thun MJ. Overweight, obesity, and mortality from cancer in a prospectively studied cohort of U.S. adults. N Engl J Med. 2003;348 (17):1625-38.

4. Raymond SU, Leeder S, Greenberg HM. Obesity and cardiovascular disease in developing countries: a growing problem and an economic threat. Curr Opin Clin Nutr Metab Care. 2006;9(2):111-6.

5. Lapidus L, Bengtsson C, Larsson B, Pennert K, Rybo E, Sjostrom L. Distribution of adipose tissue and risk of cardiovascular disease and death: a 12-year follow-up of participants in the population study of women in Gothenburg, Sweden. Br Med J. 1984;289(6454):1257-61.

6. Yusuf S, Hawken S, Ôunpuu S, Dans T, Avezum A, Lanas $F$, et al. Effect of potentially modifiable risk factors associated with myocardial infarction in 52 countries (the INTERHEART study): case-control study. Lancet. 2004;364 (9438):937-52.

7. Koning L, Merchant AT, Pogue J, Anand SS. Waist circumference and waist-to-hip ratio as predictors of cardiovascular events: meta-regression analysis of prospective studies. Eur Heart J. 2007;28(7):850-6.

8. Barbosa PJ, Lessa I, Almeida Filho N, Magalhães LB, Araújo J. Critério de obesidade central em população brasileira: impacto sobre a síndrome metabólica. Arq Bras Cardiol. 2006;87(4):407-14.

9. Grundy SM, Brewer Jr HB, Cleeman JI, Smith Jr SC, Lefant C. Definition of metabolic syndrome: report of the National Heart, Lung, and Blood Institute/American Heart Association conference on scientific issues related to definition. Arterioscler Thromb Vasc Biol. 2004;24(2):e13-8.

10. Ford ES. Prevalence of the metabolic syndrome defined by the International Diabetes Federation among adults in the U.S. Diabetes Care. 2005;28(11):2745-9.

11. Tan CE, Ma S, Wai D, Chew SK, Tai ES. Can we apply the National Cholesterol Education Program Adult Treatment Panel definition of the metabolic syndrome to Asians? Diabetes Care. 2004;27(5):1182-6.

12. Sanchez-Castillo CP, Velazquez-Monroy O, Berber A, LaraEsqueda A, Tapia-Conyer R, James WP. Anthropometric cutoff points for predicting chronic diseases in the Mexican National Health Survey 2000. Obes Res. 2003;11(3):442-51.

13. Ramachandran A, Snehalatha C, Satyavani K, Sivasankari S, Vijay $\mathrm{V}$. Metabolic syndrome in urban Asian Indian adults: a population study using modified ATP III criteria. Diabetes Res Clin Pract. 2003;60(3):199-204. 
14. Steering Committee of the Western Pacific Region of the World Health Organization and the International Obesity Task Force. The Asia-Pacific perspective. Redefining Obesity and its treatment. Health Communications Australia Pty Ltd.; 2000.

15. Grundy SM, Cleeman JI, Daniels SR, Donato KA, Eckel RH, Franklin BA, et al. Diagnosis and management of the metabolic syndrome: an American Heart Association/National Heart, Lung, and Blood Institute Scientific Statement. Circulation. 2005;112(17):2735-52.

16. Pitanga FJ, Lessa I. Indicadores antropométricos de obesidade como instrumento de triagem para risco coronariano elevado na cidade de Salvador-Bahia. Arq Bras Cardiol. 2005;85(1):26-31.

17. Benseñor IM, Latufo PA. Estado atual do tratamento e controle do diabetes melito, da dislipidemia e da hipertensão arterial no Brasil e no mundo. In: Risco cardiovascular global. São Paulo: Lemos Editorial; 2002. p.35.

18. Silber S, Albertsson P, Avilés FF, Camici PG, Colombo A, Hamm E, et al. Guidelines for percutaneous coronary interventions. The Task Force for Percutaneous Coronary Interventions of the European Society of Cardiology. Eur Heart J. $2005 ; 26(8): 804-47$.

19. Expert Panel on Detection, Evaluation, and Treatment of High Blood Cholesterol in Adults. Executive Summary of the Third Report of the National Cholesterol Education Program (NCEP) expert panel on detection, evaluation, and treatment of high blood cholesterol in adults (Adult treatment Panel III). JAMA. 2001;285(19):2486-97.

20. Hu G, Qiao Q, Tuomilehto J, Balkau B, Borch-Johnsen K Pyorala K. Prevalence of the metabolic syndrome and its relation to all-cause and cardiovascular mortality in nondiabetic European men and women. Arch Intern Med. 2004;164(10):1066-76.

21. Oh J-Y, Hong YS, Sung Y-A, Barrett-Connor E. Prevalence and factor analysis of metabolic syndrome in an urban Korean population. Diabetes Care. 2004;27(8):2027-32.

22. I Diretriz brasileira de diagnóstico e tratamento da síndrome metabólica. Arq Bras Cardiol. 2005;84(Suppl 1):1-28.

23. Rexrode KM, Carey VJ, Hennekens CH, Walters EE, Colditz GA, Stampfer MJ, et al. Abdominal adiposity and coronary heart disease in women. JAMA. 1998;280(21):1843-8.

24. Bei-Fan Z. Predictive values of body mass index and waist circumference for risk factors of certain related diseases in Chinese adults: study on optimal cut-off points of body mass index and waist circumference in Chinese adults. Cooperative Meta-Analysis Group of Working Group on Obesity in China. Asia Pac J Clin Nutr. 2002;11(suppl 8):S685-93.

25. Lin WY, Lee LT, Chen CY, Lo H, Hsia HH, Liu IL, et al. Optimal cut-off values for obesity: using simple anthropometric indices to predict cardiovascular risk factors in Taiwan. Int J Obes Relat Metab Disord. 2002;26(9):1232-8.

26. Snehalatha C, Viswanathan V, Ramachandran A. Cutoff value for normal anthropometric variables in asian Indian adults. Diabetes Care. 2003;26(5):1380-4.

27. Ito H, Nakasuga K, Oshima A, Maruyama T, Kaji Y, Harada $M$, et al. Detection of cardiovascular risk factors by indices of obesity obtained from anthropometry and dual-energy Xray absorptiometry in Japanese individuals. Int J Obes Relat Metab Disord. 2003;27(2):232-7. 